\title{
Uncovering Innovation Practices and Requirements in Privacy and Cyber Security Organisations: Insights from IPACSO
}

\author{
Zeta Dooly, Kenny Doyle ${ }^{1}$ and Jamie Power $^{2} *$ \\ ${ }^{1}$ TSSG, Waterford Institute of Technology, Waterford, Ireland z zdoolyetssg . org / \\ kdoyle@wit.ie\} \\ ${ }^{2}$ RIKON, Waterford Institute of Technology, Waterford, Ireland \{jrpower@wit.ie\}
}

\begin{abstract}
A pressing challenge facing the cybersecurity and privacy research community is transitioning technical R\&D into commercial and marketplace ready products and services. Responding to the need to develop a better understanding of how Privacy and CyberSecurity (PACS) market needs and overall technology innovation best-practice can be harmonized more effectively the contribution of this paper is centred upon uncovering PACS stakeholders' innovation practices, requirements, and challenges and in doing so highlighting scope for innovation intervention supports. The research outputs impacts and has implications at various levels, most notably in terms of framing both innovator and firm-level innovation requirements within the PACS domain, which has relevance to academic and policy making audiences also. Additionally, given that the research outputs form a pivotal component of the IPACSO project, they will actively contribute to ongoing debates and objectives around shaping support measures for PACS innovation awareness, competency building and innovation policy support developments in the domain.
\end{abstract}

Keywords: innovation, requirements, challenges, privacy, cybersecurity

\section{Introduction and Research Focus Rationale}

The publication of the EU CyberSecurity Strategy [1] coupled with Europe 2020 strategy and its flagship initiatives such as The Innovation Union and Digital Agenda all underscore the escalating importance of innovation. Reflective of this, opportuni- 
ties for innovators in the privacy and cybersecurity domain is increasing. Nonetheless, challenges of transitioning technology related research developments and outputs to real-world deployment are well documented. Nonetheless, a range of challenges including, but not limited to: pursuing a narrow innovation process failing to incorporate the internal and external ecosystem or customer needs, an overemphasis on technology-driven bottom-up innovation, in addition to unsupportive deployment channels for research output/commercialization's hamper the transitioning of technology related research developments and outputs to commercial deployment [2].

A pressing challenge facing the cybersecurity and privacy research community is transitioning technical R\&D into commercial and marketplace ready products and services - "New and innovative technologies will only make a difference if they are deployed and used. It does not matter how visionary a technology is unless it meets the needs and requirements of customers/users and it is available as a product via channels that are acceptable to the customers/users" [2]. While innovation is widely recognized by industry and academics as a sustainable and competitive enabler, nonetheless understanding of innovation management and practice remains fragmented, misunderstood and untamed by practitioners and researchers [3] [4]. Innovation practice and requirements are far from straightforward "...most innovation is messy, involving false starts, recycling between stages, dead ends and jumps out of sequence" [10]. Varying attempts have been made to articulate conceptual order on the innovation processes of organisations, in the form of innovation process models and the variety amongst the models is the consequence of a lack of consensus as to how an innovation process should look like, given the unique requirements, contexts, environments, and purposes for which they are developed [10]; [11]. Indeed, several authors acknowledge that innovation process does not occur within a vacuum, and thereby indicate a range of contextual factors which impact on the processes deployed [12]; [13]; [14]; [15]. Such contextual factors range from organisational characteristics to societal factors and from influenceable factors to external factors.

Innovators operate within complex and turbulent environments, and are increasingly confronted with escalating and rapid technology developments, competitive global market competition and shorter product life cycles meaning they must be reactive and flexible to organizational, technological and market shifts [5]. Indeed, the privacy and cybersecurity market is deeply influenced from various themes driven by technical, human, societal, organizational, economic, legal, and regulatory concerns among others; these factors combine to create marketplace and innovation ecosystem with complex value chain relationships [6]. Innovation therefore cannot not occur within a vacuum and is impacted upon by a range of external contextual factors in addition to the following internal considerations, including but not limited to, strategy and culture, resources and skills, leadership, organizational structure and external linkages [7], [8], [9]. Reflective of the above, innovation practice is far from straightforward “...most innovation is messy, involving false starts, recycling between stages, dead ends and jumps out of sequence” [3]. 
Mindful of this, through a specific PACS lens, IPACSO aims to support innovators in both industry and research communities with a responsive innovation framework to enhance their overall innovation engagement, management and deployment activities. IPACSO is an EU-funded Coordination and Support Action (CSA) project aimed at supporting Privacy and CyberSecurity innovations in Europe www.ipacso.eu. IPACSO is focused on adapting existing innovation methodologies available in other domains, both general and specific; optimizing these approaches for the Privacy and CyberSecurity (PACS) market domains. The research outputs impacts and has implications at various levels, most notably in terms of framing both innovator and firm-level innovation requirements with reference to informing the IPACSO framework. Additionally, given that the research outputs form a pivotal component of the IPACSO project, they will actively contribute to ongoing debates and objectives around shaping support and policy measures for PACS innovation awareness, competency building and innovation policy support developments in the domain.

\section{Research Methodology}

In pursuit of identifying PACS stakeholders' innovation requirements a small-scale mixed method triangulated research design was employed, encompassing an online questionnaire, semi-structured telephone interviews and secondary desk research. Derived from IPACSO's overarching stakeholder focus, two key categories of interest formed the target sampling frame; specifically innovators and enablers. "Innovators": individuals or companies that are looking to bring ideas in the PACs domain to market. Sub-categories include researchers, vendors, service providers, integrators and infrastructure providers. "Enablers": individuals or entities who are responsible for supporting individuals or companies in being more innovative and in commercialising technology. The research respondents included IPACSO members, Innovation Advisory Board Members, NIS WG3 members amongst other individuals and organisations engaged with through IPACSO exploitation and dissemination events.

This triangulated research design approach enabled for multiple sources of data to be collected and integrated in pursuit of documenting stakeholders' innovation requirements and enhancing the reliability and validity of the subsequent analysis. The survey design, which consisted of ranking and open ended questions was informed from the Community Innovation Survey guide and was administered online via SurveyMonkey. A semi-structured interview guide was developed in parallel to the survey instrument.

Reflecting IPACSO's multi-stakeholder foci, a broad range of stakeholder categories are represented in the research findings ranging from industry innovators in the PACS domain, research innovators, innovation intermediaries in the form of consultancy and industry support, in addition to funding and policy representatives. PACS 
relevant subdomains of those who participated in the research include but are not limited to: mobile and cloud security, telco, cyber protection, cryptography, malware, privacy enhancing technologies, surveillance and intrusion detection, security intelligence, distributed computing and big data. Regarding organisation size, categories ranging from micro to large are represented with small organisations (34.8\%) leading the response rate followed by micro $(26.1 \%)$ and large $(26.1 \%)$ and medium size organisations $(13 \%)$ respectively. The data reflects the growing consensus of small enterprises proliferating the diverse and fragmented PACS landscape, with small and micro firms accounting for over half of all participants in the research. Demonstrating a diverse canvas of participation from all areas within organisational structures, respondents included: founders and directors, R\&D managers and personnel, CTO's, commercial directors and business developers, CEO's, project and product managers, technology transfer managers, professors and researchers from research institutes, policy makers and security evangelists.

\section{$3 \quad$ Research Findings}

This research findings are focused on the innovation practice of PACS stakeholders, in the context of the innovation value chain, and serves to provide requirements and scenario inputs to inform the development of the IPACSO Innovation Framework. For this reason, the primary research investigation focused on identifying stakeholders innovation scenarios, practices and requirements to develop an understanding of the following:

- Stakeholders' innovation practices, including current environment, approaches and requirements in relation to innovation engagement.

- Challenges, barriers and support requirements in relation to PACS innovation.

\subsection{Innovation Practices}

\section{- A diverse approach to organising innovation transcends the domain}

While two thirds of respondents indicated that an innovation strategy(s) is in place in their organisation, the survey revealed that there are variances in terms of supporting and complementary policies and procedures underpinning such strategies.Equally, a broad range of processes for organising innovation were collected from the respondents. Two thirds of respondents adopt a cross functional approach to facilitate innovation; whereas a third utilize specialized organization units (e.g. research centres). Of note, over a quarter of respondents reported an ad-hoc, informal approach to innovation organisation and a further $16.7 \%$ identified that their innovation operations are conducted externally through outsourcing arrangements. 


\section{- Multi-disciplinary internal and external stakeholder involvement}

A wide-ranging spectrum of stakeholders are involved in innovation activities, albeit at varying levels. Internal staff represent the highest frequency of stakeholders used, followed by a combination of clients/customers, competitors, consultants are utilised at lower levels of frequency with professional/industry associations, universities and government/research institutes being used as less frequent partners. A significant proportion of respondents indicated that external stakeholders such as suppliers, competitors and consultants are never involved in the innovation processes or activities within their organisation.

\section{- Multiple and integrated innovation models are utilised}

Demonstrating that innovation practice is a combination of technology push and demand pull dimensions, both of these categories are strongly represented amongst the respondents. Reflecting the previously reported dominant role of internal cross functional staff integration, a cooperative and parallel approach is also commonly pursued. Indicating a potential lack of innovation governance, only one in in five respondents reported a stage gated process. Underscoring the escalating incidences of collaborations between innovating organisations and external stakeholders, over $50 \%$ positive agreement statements were reported for systems/networking integration and open innovation models.

\section{- Product and service innovation are primary foci}

In terms of the respondents to this study product and service innovation dominate their primary innovation focus; whereas process innovation represents the key secondary focus. Conversely, organisational and marketing innovation was not reported as a focus by $50 \%$ and $40 \%$ of respondents respectively.

\section{- Innovation competency levels vary across the innovation value chain}

When asked to rate their level of competency across the various phases of an innovation lifecycle the respondents identified high and competent levels of proficiency is the areas of ideation and concept development and design and business analysis. Nonetheless, it was still reported that ideation and business analysis phases lacked systematic and comprehensive attention (Figure 11). On a more positive note, almost $80 \%$ of respondents identified that their development processes are flexible enough to be adapted to market conditions and project reports. Indeed, over half of responses identified that lean and agile approaches are followed for innovation development. Regarding the concluding aspect of the innovation process, i.e. the launch, less than half of respondents utilize a multi-disciplinary team approach to ensure their innovation outputs are targeted, launched and delivered to the marketplace. Areas where respondents felt there was scope for improvement included the phases towards the end of the lifecycle including test, implementation and post launch. 
- Innovation practice and requirements vary by the maturity levels of Organisations.

Echoing the WEF fostering innovation report [20] which categories entrepreneurship driven innovation into three categories - stand up, start up, scale up - the level of innovation practice and requirements of innovators varies depending on their respective maturity level. For instance, respondents from MNCs identified that broad, complex and highly structured innovation ecosystems, departments, policies and strategies are a hallmark of their organisations. Such infrastructures accordingly facilitate a complex web of innovation activities both internally and externally encompassing industrial applied research projects, technology driven research and collaboration with other companies and research institutes/universities. The reported positives of such an environment included the access of multi-disciplinary support from internal stakeholders to develop both technical and business case advances. It was reported that large MNC operations have dedicated resources, facilities and manpower to consistently and systematically scan for external innovations that may be capable of exploitation. Examples include: monitoring start-ups, incubators/labs, competitions for SMES, Hothouse Brainstorming sessions, funding research programmes centres in universities, collaboration with SMEs. Equally so, negatives were reported in relation to an overly bureaucratic, stage-gated innovation environment and infrastructure with reference to research project lags versus short time market opportunities "Frameworks are difficult too - they can be a straitjacket or an enabler". Conversely, small scale start-up respondents reported that their relative infancy in terms of maturity restricted their capacity to implement and deploy defined and structured innovation systems; largely due to financial, manpower and access to networking constraints - " ...if you are a start-up you need to factor in overheads to go through a process. Often start-ups favour getting bought up by larger companies in order to fully realise and exploit their idea/concept".

\section{- Value chain positioning impacts on innovation focus}

The majority of observable innovation in cyber-security and privacy markets is best described as incremental. This means that much of the innovation is a product or service improvement, but not a radically new development that forces businesses to re-organization or leads to the emergence of wholly new markets. For instance, a responding Telco organisation identified that given their positioning in the middle of the supply chain, their innovations are incremental in terms of integrating components of technology from suppliers, tech plug-ins for a platform or providing a service wrap around technology deliver. In a similar vein, a software services, devices and solutions company reported that that they do not produce many classic cyber security products. Instead, they strive that cyber security is built in to products and services as their customers expect that what they deliver is secure. 


\subsection{Innovation Challenges}

the challenges and barriers to innovation are well documented: understanding of innovation management and practice remains fragmented, misunderstood and untamed by practitioners and researchers [16]; [17]; [18]; innovators operate within complex and turbulent environments, and are increasingly confronted with escalating and rapid technology development, global market competitive and shorter product life cycles requirements [19]; innovation is impacted upon by a range of external contextual factors in tandem to internal considerations, including but not limited to, strategy and culture, resources and skills, leadership, organizational structure and external linkages [12]; [14].Reflective of the above the respondents were asked to rate how the following typical innovation challenges related to their organisation. The typical innovation challenges included:

- Infrastructure Factors (e.g. lack of innovation governance, inadequate innovation management procedures, ad-hoc R\&D practices, lack of collaborative structures etc.);

- Cost Factors (e.g. lack of appropriate funds within the enterprise/ from external sources, innovation costs too high etc.);

- Knowledge Factors (e.g. lack of qualified personnel, limited information on markets, difficulty in finding cooperation partners etc.);

- Market Factors (e.g. market dominated by established enterprises, uncertain demand for goods and services etc.);

- Legal and Regulatory Factors (e.g. escalating legislative and regulatory requirements)

As presented in Figure 1, variance was reported across all categories of challenge factors. Unsurprisingly, cost factors came first for all the respondents with a score in the region of $70 \%$. One out of five respondents also identified knowledge and market factors as a serious problematic innovation challenge. A significant finding is that all of the challenge factors rated as both moderate and minor challenges for the respondents.

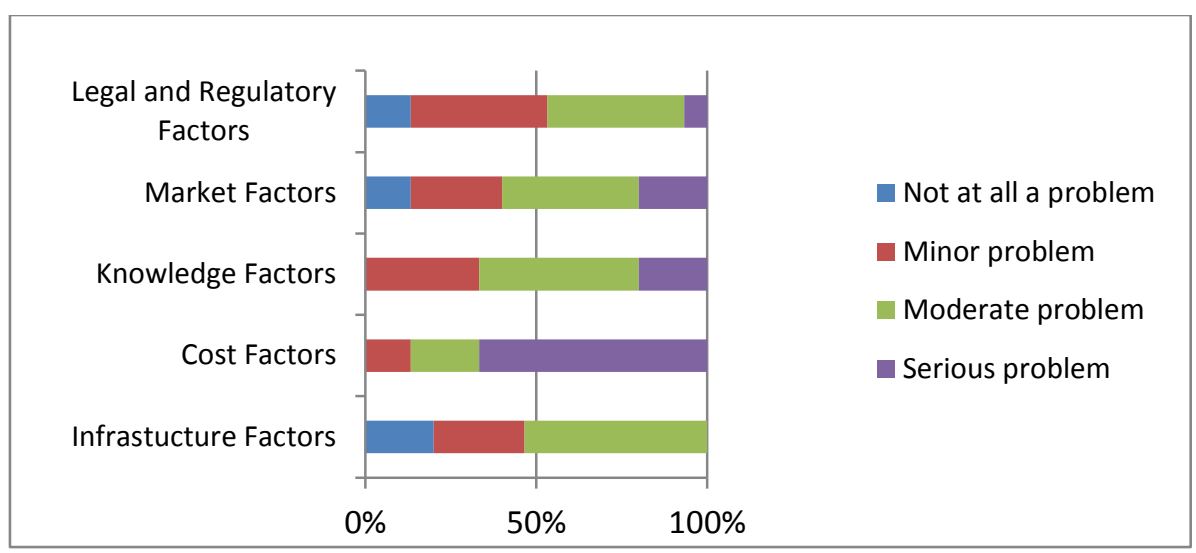




\section{Figure 1 Innovation Challenges}

Elaborating upon these findings, Table 1 below synopsizes a range of related and additional challenges which impede undertaking innovation in the PACS context. Replicating the findings in Figure 1 above, cost, regulatory, infrastructure and market forces are represented, in addition to business knowledge, threats, awareness and acceptance challenges.

\begin{tabular}{|l|l|}
\hline $\begin{array}{l}\text { Human (skills, intel- } \\
\text { ligence, availability) }\end{array}$ & $\begin{array}{l}\text { Very high expertise of internal resources. Access to the right develop- } \\
\text { ers with specialised competence/ Skilled resources. Idea implement- } \\
\text { ers. Staff shortages }\end{array}$ \\
\hline Funding/ resources & $\begin{array}{l}\text { Financial resources/ funding (we operate 100\% on cash flow).Cost of } \\
\text { development. Competing internal resources }\end{array}$ \\
\hline Policies/ procedures & $\begin{array}{l}\text { Internal practices. Common policies missing. IPR and patent land- } \\
\text { scaping }\end{array}$ \\
\hline Market issues & Competitiveness between collaborators. Market positioning issues \\
\hline Regulation & $\begin{array}{l}\text { Regulatory barriers. Navigating the minefield. Stumbling block. De- } \\
\text { tect, block and clean new malware }\end{array}$ \\
\hline Business Knowledge & $\begin{array}{l}\text { Business modelling. Underpinning business case. Diffusion and route } \\
\text { to market }\end{array}$ \\
\hline $\begin{array}{l}\text { Awareness and } \\
\text { Acceptance }\end{array}$ & $\begin{array}{l}\text { Acceptance of new technology concepts. Education in privacy en- } \\
\text { hancing technologies }\end{array}$ \\
\hline Top management & Corporate engagement and involvement \\
\hline
\end{tabular}

Table 1 Innovation Barriers in PACS

\section{- PACS trends constantly move the goalposts}

While market shifts and demands represent a key innovation component and driven in any industry setting, the constantly changing and hard to predict PACS environment exerts a significant challenge. Interview respondents were in agreement that the speed of innovation and short product cycles are signature aspects of digital markets which are continuously altered through emerging threat and vulnerabilities "it's a continuous race between hackers and solution, the target is always moving and so too is the risk". The analogy of a Knight in a Suit of Armour was used to describe the imperative of being able to move and fight in terms of innovation engagement. Equally so, it was cautioned that research, innovation and development priorities cannot be solely based on today's problem - the world moves on, new waves of technology and threats are emerging, the key is finding windows of opportunity.

\section{- Difficult to retro-fit Privacy and Cyber Security innovation focus}

A significant proportion of the interview respondents signaled that in order for innovation outcomes to be successful in the domain, PACS specific guiding principles 
should be a motivator, as opposed to an afterthought of product/service development. "It is much more difficult to retro-engineer at the end, security is all about how it is used and should be a driving force from concept commencement". This point was also echoed in relation to privacy specific innovation applications "privacy is given little attention in the design phase"; however it was noted that privacy by design was gaining traction as a value proposition in terms developing technologies that are respectful of data protection, privacy legislation.

\section{- Bureaucratic funding/support mechanisms}

The interview respondents who have current and previous experience of participating in both national and European innovation funding initiatives reported frustrations and concerns surrounding such instruments in light of the fast paced, short lifecycle demands of the PACS environment. Some argued the typical three year timeframe was too restrictive in terms of getting products/solutions to close to market stage; whereas others argued that projects should be longer to accommodate the early stages of the innovation value chain lifecycle. It was recognised that with the advent of Horizon 2020 , concentrated efforts were being mobilized to facilitate more agile innovation activities and a broader spectrum of funding criteria with reference to innovation actions.

\subsection{Innovation Requirements}

When questioned about innovation areas/aspects where they consider support, guidance and knowledge would be of benefit the respondents highlighted a range of requirements and scope for opportunities. Figure 2 presents the results, and indicates essential and high priorities across the board in all of the areas with between $25 \%-65$ $\%$ of respondents. Strong requirements for innovation supports were reported in the areas of portfolio management, post launch, resource and competence management and business intelligence. Elaborating upon these findings, Table 3 synopsizes a range of related and additional innovation requirement areas, in terms of areas presenting scope for improvement. Replicating the findings in Figure 2 above, cost, market, human and business intelligence are strongly priority in addition to calls for networking, collaboration and innovation/risk awareness building. 


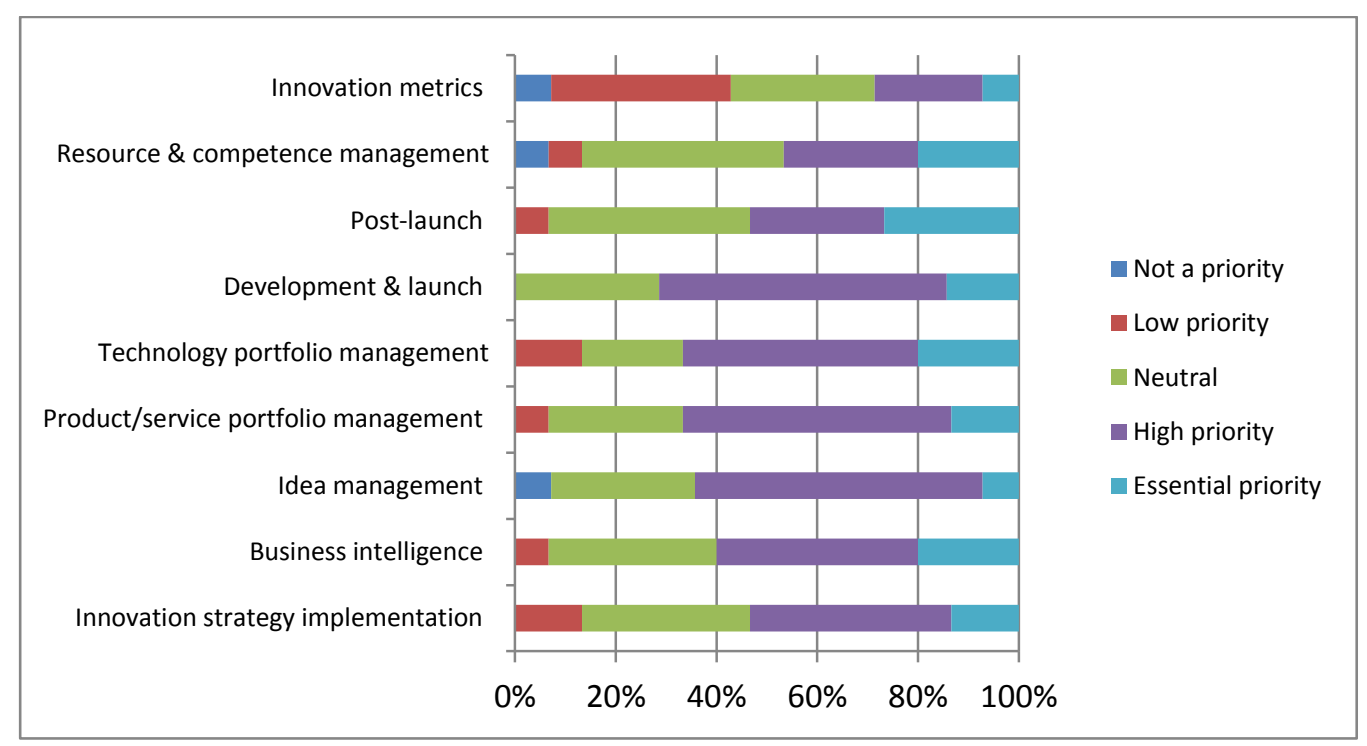

Figure 2 Scope for Innovation Supports

\begin{tabular}{|l|l|}
\hline Economic Supports & $\begin{array}{l}\text { Funding of expensive projects; EU/Government incentives in inno- } \\
\text { vation (Tax incentives); Economic assistance and investment sup- } \\
\text { ports. }\end{array}$ \\
\hline $\begin{array}{l}\text { Networking and Col- } \\
\text { laboration supports }\end{array}$ & $\begin{array}{l}\text { Assistance in linking with major companies; Programmes to encour- } \\
\text { age smaller and larger companies to collaborate. }\end{array}$ \\
\hline Market Supports & $\begin{array}{l}\text { Regulation screening and patent searching; Targeted initiatives } \\
\text { aimed at channel development; Assistance in scanning the market; } \\
\text { Resources for market knowledgebase identification, needs identifi- } \\
\text { cation. }\end{array}$ \\
\hline $\begin{array}{l}\text { Human/People sup- } \\
\text { ports }\end{array}$ & $\begin{array}{l}\text { Top management commitment; Access to key competence for hir- } \\
\text { ing; dedicated training and consultancy supports. }\end{array}$ \\
\hline $\begin{array}{l}\text { Business Development } \\
\text { Supports }\end{array}$ & $\begin{array}{l}\text { Market positioning; Marketing; Business intelligence; PR; Imple- } \\
\text { mentation and customer engagement; Benchmarking. }\end{array}$ \\
\hline $\begin{array}{l}\text { Risk and Awareness } \\
\text { Building Supports }\end{array}$ & $\begin{array}{l}\text { Initiatives for encouraging disruptive innovation engagement; Con- } \\
\text { fidence building in ideation and follow through; Initiatives to pro- } \\
\text { mote European enterprises to be leaders as opposed to followers. }\end{array}$ \\
\hline
\end{tabular}

Table 3 Additional Scope for Innovation Supports

- Importance of marrying business, technology and research excellence

A common denominator from the interview findings is the varying levels of disconnect between research and technology development and innovation diffu- 
sion/implementation. While the imperative of underpinning innovation development activities with sound commercial business cases was recognised by all, competency and proficiency in this area varies significantly. This was particularly pronounced in an interview with a business development manager within a university cyber security research group - "....commercial validation, demand and risk is not well understood by researchers; technologists don't focus on intricacies of business modelling or marketplace risk".

\section{Concluding Remarks}

A diverse range of innovation modelling processes, practices and, in turn, requirements proliferate the PACS innovation domain. The analysis, which triangulates survey, interview and desk research, indicates a diverse and varied perspective of innovation organisation and practice in the PACS domain.

- Multiple and integrated innovation models are utilised which draw upon elements of technology push, demand pull, cooperative, networking and open innovation principles. This variance, creates difference scenarios of practice and focus both in terms of the stakeholders involved and the phases/gates deployed and in turn, their requirements.

- The level of innovation practice and requirements of innovators varies depending on their respective maturity level. While market shifts and demands represent a key innovation component and driver in any industry setting, the constantly changing and hard to predict PACS environment exerts a significant challenge.

- At a high level, the research indicates that existing competencies and investment are directed in the early phases of the innovation lifecycle (ideation through to concept development); whereas significant scope and requirements occur in the latter stages (test and implementation).

- A significant finding is that innovation challenges transcend infrastructural, market, knowledge, cost and legal domains. Cost factors came first for all the respondents with knowledge and market factors also representing a serious problematic innovation challenge.

- The stakeholders identified a broad scope for innovation supports across the entire innovation value chain and ecosystem (i.e. strategy, business intelligence, ideation, portfolio management, resource management development, and launch). A common denominator from the interview findings is the varying levels of disconnect between research and technology development and innovation diffusion/implementation. While the imperative of underpinning innovation development activities with sound commercial business cases was recognised by all, competency and proficiency in this area varies significantly.

Turning to recommendations gleaned from the analysis, the research outputs impacts and has implications at various levels, most notably in terms of framing both 
innovator and firm-level innovation requirements within the PACS domain, which has relevance to academic and policy making audiences also. Additionally, given that the research outputs form a pivotal component of the IPACSO project, they will actively contribute to ongoing debates and objectives around shaping support measures for PACS innovation awareness, competency building and innovation policy support developments in the domain.

- For innovators - it is pertinent to note that there is no one size fits all solution to designing and implementing a successful innovation process as each innovation ecosystem and value chain needs to be aligned to its respective organisational context. Nonetheless, there is an ever increasing general body of information around innovation practice and modelling which has direct relevance to informing firm-level innovation practice: i.e. the set of rules, models and stages involved; considerations for R\&D, utilizing both internal and external knowledge sources/collaborators and responding to market forces and the strengths and weaknesses of the various generations of innovation models.

- For policy makers and enablers - the analysis highlights the importance of the need to integrate the innovation ecosystem (internal and external) and consider the various stages of the innovation lifecycle/value chain in terms of supporting and cultivating end-to-end innovation activities. Innovation is more than the technical output (irrespective if that output is product or service orientated) and interventions at policy and enabling levels need to adapt and/or continue to prioritise infrastructural, ecosystem, and 'soft' people related initiatives and actions to ensure a balanced innovation support offering.

- For IPACSO Innovation Framework - the respective outputs of the survey and interview data will directly input into shaping the core and supporting innovation modules The actual components and content of the IPACSO framework will, in turn be developed into decision support modules and associated toolkits which will be equally iteratively developed, trialed and validated with target stakeholder engagement, primarily through validation training Bootcamps and wider dissemination and outreach channels.

Furthermore, the research insights, and the IPACSO project overall, will have relevance to the European trust and security Framework research programme portfolio which are increasingly charged with focusing on potential innovation arising from their activities, in terms of increasing project outputs for economic and societal benefit.

\section{Bibliography}


[1] EC, "Cyber Security Strategy of the European union: An Open Safe and Secure Cyberspace," 2013.

[2] D. Maughan, D. Baleson, U. Lindqvist and Z. Tudor, "Crossing the "Valley of Death": Transitioning Cybersecurity Research into Practice," Journal IEES Security and Privacy, vol. 11, no. 2, pp. 14-23, 2013.

[3] J. Tidd, "A Review of Innovation Models Discussion Paper 1," Science and Technology Policy Research Unit, Tanaka Business School, University of Sussex, 2006.

[4] Z. Dooly, S. Galvin, J. Power, B. Renard and U. Seldeslachts, "IPACSO: Towards Developing an Innovation Framework for ICT Innovators in the Privacy and cyberSecurity Markets," in Cyber Security and Privacy Third Cyber Security and Privacy EU Forum, CSP Forum 2014, , Springer, 2014, pp. 148-158.

[5] R. Garud, A. Kumaraswamy and V. Sambamurthy, "Emergent by Design: Performance and Transformation at Infosys Technologies," Organizational Science, vol. 1, no. 277, 2006.

[6] OSMOSIS, "D2.1 Report on the Identified Security's Market Potential/ D2.2 Report on Taxonomy Definition," http://www.osmosisecurity.eu/system/files/OSMOSIS_D2.1\%20and\%20D2.2_int egrated.pdf, 2010.

[7] R. Rothwell, "Towards the Fifth-Generation Innovation Process," International Marketing Review, vol. 11, no. 1, pp. 7-31, 1994.

[8] K. Cormican and D. O'Sullivan, "Auditing Best Practice for Effective Product Innovation," Technovation, vol. 24, no. 10, pp. 819-829, 2004.

[9] D. Jacobs and H. Snijders, "Innovation Routine: How Managers can Support Repeated Innovation," Stitching Management Studies, Van Gorcum, Assen, 2008.

[10] J. Tidd, "A review of innovation models discussion paper 1," Science and Technology Policy Research Unit, Tanaka Business School, University of Sussex, 2006.

[11] C. Eleveens, "Innovation Management: A Literature Review of Innovation Process Models and their Implications," Nijmegen, NL, 2010.

[12] R. Rothwell, "Towards the Fifth-Generation Innovation Process," International Marketing Review, vol. 11, no. 1, pp. 7-31, 1994.

[13] A. Van de Ven, H. Angle and M. Poole, Research on the Management of Innovation: The Minnesota studies., New York: Harper \& Row, 1989.

[14] K. Cormican and D. O'Sullivan, "Auditing Best Practice for Effective Product Innovation Management," Technovation, vol. 24, pp. 819-829, 2004.

[15] J. Tidd, J. Bessant and K. Pavitt, Managing Innovation - Integrating Technological, Market and Organizational Change, New York: John Wiley \& Sons, 2005.

[16] R. Wolfe, "Organizational innovation: review, critique, and suggested research directions," Journal of Management Studies, vol. 31, pp. 405-431, 1994. 
[17] R. Drazin and C. Schoonhoven, "Community, Population, and Organization Effects on Innovation: a multilevel perspective," Academy of Management Journal, vol. 39, pp. 1065-1083, 1996.

[18] J. Tidd, "Innovation Management in Context: Environment, Organization and Performance," International Journal of Management Review, vol. 3, no. 3, pp. 169-183, 2001.

[19] R. Garud, A. Kumaraswamy and V. Sambamurthy, "Emergent by Design: Performance and Transformation at Infosys Technologies," Organizational Science, vol. 17, no. 2, pp. 277-286, 2006.

[20] World Economic Forum (WEF), "Enhancing Europes CompetitivenessFostering Innovation Driven Entrepreneurship in Europe," http://www3.weforum.org/docs/WEF_EuropeCompetitiveness_InnovationDriven Entrepreneurship_Report_2014.pdf, 2014. 